\title{
The effect of secondary reinforcement on differential instrumental conditioning in rats'
}

\author{
JAMES R. ISON, UNIVERSITY OF ROCHESTER \\ WILLIAM CLAIBORN, SYRACUSE UNIVERSITY
}

A food cup placed in S- maintained response strength to that stimulus, confirming the hypothesis that secondary reinforcement retards discrimination learning because of its effect on the incorrect response. No effects were noted on $S_{+}$or on speeds to the old $S_{-}$in a subsequent reinforcement stage.

If a secondary reinforcing stimulus follows an incorrect response in simultaneous discrimination learning then the formation of the discrimination is retarded (Denny, 1948; Ehrenfreund, 1949). Most simply this effect can be related to studies of experimental extinction which demonstrate that secondary reinforcement attenuates the effect of non (primary) reinforcement. If it is assumed that discrimination learning depends in part on the extinction of the incorrect response and, further, that this follows the same laws as found in simple experimental extinction, then it follows that the retarding effect of secondary reinforcement on discrimination learning is produced by its maintaining the strength of the incorrect response. This hypothesis, which has some general acceptance (Kimble, 1960, p. 192) was put to a direct test using a differential instmumental conditioning procedure, as part of a set of experiments investigating the transition from simple conditioning and extinction to discrimination learning.

In addition to the major hypothesis we were sensitive to two other potential effects. First, it was possible that in the discrimination situation there might exist a complex interaction such that the secondary reinforcement of the incorrect response had an effect on the strength of the correct response. Second, it was possible that the stimulus might produce eventually greater aversion such that the terminal level of incorrect performance would be reduced. In anticipation of the results, the major hypothesis was confirmed but neither of the two potential effects were observed. Subjects

The Ss were 32 male albino rats, 90 days old, from Holtzman, Madison, Wisconsin.

\section{Apparatus}

The differential conditioning box consisted of two parallel alleyways, 36 in. long, $3-1 / 2$ in. wide and 4 in. high, to each of which a start box, 12 in. long, $3-1 / 2$ in. wide and 4 in. high could be attached. Each section was covered with Plexiglas. The start box and its side-action retrace door were painted mid grey, one alley a flat black and one a flat off-white. Baffles at the alleyends concealed a drilled aluminum block which held .20 cc of liquid. Photo cells, on the start door and 12 in. down the alleys, controlling a Hunter Klockounter, recorded start times.

\section{Procedure}

The Ss had water available for $1 \mathrm{hr} \cdot 10-15 \mathrm{~min}$. after the daily treatment (food ad lib). They were placed on this schedule and individually handled for 2 min. each day for five days before training. One pair of trials was given on Day 1, two on Day 2 and three per day thereafter. The first pair of daily trials began with $S+$ and the order of $S+$ and $S-$ in the two remaining pairs was random. Intertrial intervals were approximately $10 \mathrm{~min} .$, and Ss were detained in S- for $8 \mathrm{sec}$.

Half of the Ss had the empty aluminum food cup on S-, and half had no food cup. In each group half were trained with $\mathrm{B}+, \mathrm{W}-$, half the reverse.

Stage 1 continued for 96 trials, 48 to each stimulus. Thereafter $S s$ in replication $1(N=20)$ had the stimulus reinforcement contingencies reversed (Stage 2A) and $\mathrm{Ss}$ in replication $2(\mathrm{~N}=12)$ were reinforced on both stimuli (Stage 2B). Stage 2 continued for 15 trials to each stimulus.

\section{Results and Discussion}

Group results are in Fig. 1. Secondary reinforcement in Stage 1 had an obvious effect on the strength of the incorrect response (for the Groups by Trials interaction $F=1.91$, $d f=47 / 1316, p<.01$ ), but no effect on the correct response $(p>.20)$. The effect was confined to the intermediate trials on $\mathrm{S}-$, and there seemed to be no greater terminal decrement associated with secondary reinforcement. Median trials to

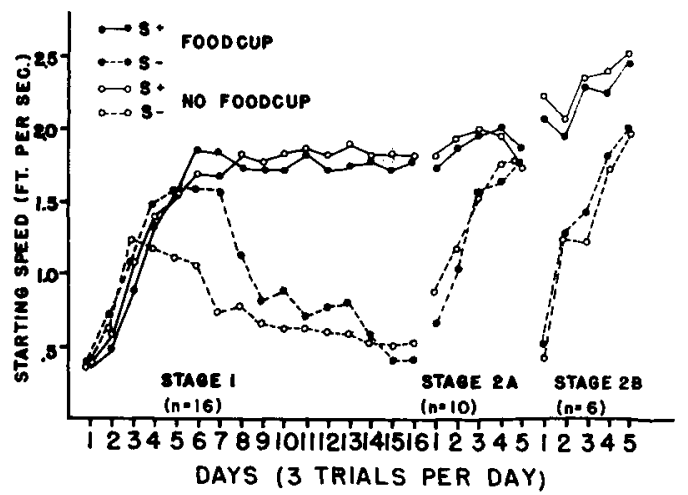

Fig. 1. Starting speed to $S+$ and $S-$ in initial acquisition (Stage 1) and subsequent reversal (S tage $2 A$ ) or nondifferential reinforcement (Stage 2B). 
a criterion of six trials without overlap was 15 with no secondary reinforcement, and 24 with secondary reinforcement $(p<.05)$.

In Stage 2 there was no effect of the food cup on either the prior S+ or the prior S-. The latter observation indicates that there was no differential inhibition associated with the old S-. Additionally, there was no overall significant effect on speeds to the prior S- of whether the prior St was or was not non-reinforced; however it appeared that a difference was developing on the last day of Stage 2. With either reward or non-reward of the prior $S+$ there was an increase in speed to that stimulus in the second stage, which was observed in each $\mathrm{S}$; this increase was greater if that stimulus continued to be associated with reward. The increase in speed to the $S+$ that was now shifted to zero reward in Stage 2 is potentially interesting, and might be seen as an exciting effect of non-reward. However in other differential conditioning experiments in which Stage 2 was an extinction phase we have not seen this increase. Most probably, then, it simply represents a condition in which the generalized excitatory effects from the other stimulus were greater than the developing inhibitory effects on the new negative stimulus.

The group curves reveal a gradual increment in excitatory strength on S+ and, following a period of trials, a gradually developing inhibition on $\mathrm{S}-$, in which secondary reinforcement modulates the growth of inhibition. However, in this experiment the behavior of no single $\mathrm{S}$ was adequately represented by the group curve. In marked contrast, the individual changes in behavior exhibited patterns which were almost entirely obscured in the summary data. Four stages can be roughly described in the individual S. First, there was an initial increase in speed to both stimuli, with S- speeds above S+ speeds. Second, there followed a small non-differential decrement in speed to each stimulus. Third, there was an abrupt large decrement in speed to S-. For about $25 \%$ of the animals this decrement remained thereafter whereas for others one or more trials occurred with fast and slow speeds interspersed. Fourth, there was a gradual recovery in speed to St. The last three stages were obscured in grouped data by Ss' entering the second stage on different trials. Thus the secondary reinforcement effect occurred not because a gradual growth of inhibition was altered in slope, but because Ss broke at different trials. The terminal level after the break seemed unaffected by the presence of the food cup.

This abrupt appearance of differential responding is similar to that observed by Krechevsky (1932) in simultaneous discrimination learning, an observation that did much to prompt the continuity-noncontinuity argument in discrimination learning. Krechevsky's explanation was that the organism shifted from a spatial to a visual hypothesis. Similarly Spence (1936) postulated disproportionate strengths of spatial responses which overcame the gradual development of differential excitatory strength to the discriminanda. Neither of these positions is appropriate to explain the abruptness of the present discrimination because the procedure eliminated position cues and associated responses or, alternatively, spatial hypotheses.

A more critical problem raised by the individual data is the relationship between the non-differential performance in the first stage and the underlying learning process. One might suspect that the lack of differential performance represents non-differential learning; this is not the case because reversal in this initial stage of differential conditioning retards subsequent learning (Edwards, 1966). Perhaps reversal depends on the extinction of reinforced response strength whereas non-reversal (i.e., continued learning) depends on the extinction of generalized response strength. Potentially this could be a further example of the weaker resistance to extinction of generalized responses (Hoffman, 1966).

\section{References}

Denny, M. R. The effect of using differential end boxes in a simple T-maze learning situation. J. exp. Psychol., 1948, 38, 245-249.

Edwards, D. C. Effect of prior experience with discriminanda on differential conditioning. Psychol. Rep., 1966, 18, 343-349.

Ehrenfreund, D. Effect of a secondary reinforcing agent in blackwhite discrimination. J. comp. physiol. Psychol., 1949, 42, 1-5.

Hoffman, H. S. The stimulus generalization of conditioned suppression. In D. I. Mostofsky (Ed.), Stimulus generalization. Stanford: Stanford University Press, 1965.

Kimble, G. A. Hilgard and Marquis' conditioning and learning. New York: Appleton-Century-Crofts, 1961.

Krechevsky, I. "Hypotheses" in rats. Psychol. Rev., 1932, 39 516-532.

Spence, $\mathbf{K}$. W. The nature of discrimination learning in animals Psychol. Rev., 1936, 43, 427-449.

\section{Note}

1. This research was supported by NSF GB 1505 and GB 4373 to JRI, and was performed in part during the junior author's tenure as an NSF Undergraduate Research Participant.

\section{Erratum}

JONES, E. C., EDMONDS, E. M., \& MUELLER, M. R. Effects of reinforcement on retention and transfer. Psychon. Sci., 1966, 6 (4), 181-182-On p. 181, the first sentence under Results and Discussion should read: "The number of trials and the number of errors to the first errorless trial...." 